\title{
PROBLEMATIQUE DE L'EXPLOITATION MINIERE INDUSTRIELLE ET ARTISANALE DANS LE DISTRICT URBANO RURAL DE KOLWEZI
}

Par : Maitre IKONGA - KAPENDA Timothée, Assistant UNIKOL, Maitre MUKINI KASHALA Augustin, Assistant UNIKOL, KASONGO NYEMBO Marcel, Assistant ISTA et Maitre KATOLU MULANGU Junior.

\section{INTRODUCTION}

En République Démocratique du Congo, les activités minières sont régies et prévues par la loi $\mathrm{n}^{\circ}$ 007/2002 du 11 juillet 2002 portant code minier. L'histoire écrite et oral appris de nos vieux, confirme que l'exploitation des ressources naturelles particulièrement des substances minérales, date de longtemps. Et c'est depuis la nuit de temps et cela ne cesse d'attirer les investisseurs étrangers et nationaux. Les chercheurs viennent aussi de différents horizons pour étudier non seulement ces richesses mais aussi l'impact de leur exploitation sur le plan local, national, sous régional et régional eu égard au rôle que doit jouer la R.D.C. dans le concert de nations.

Certes, l'exploitation minière figure parmi les secteurs jouant un rôle important dans l'économie nationale, car depuis longtemps, l'industrie minière a contribué au produit intérieur brut et aux exportations. Il en est ainsi de l'année 1970, année au cours de laquelle elle avait contribué pour environ $50 \%$ au P.I.B. et pour $80 \%$ aux exportations exprimées en valeur. Pour la même année, la contribution des entreprises minières s'élevait à 44,5\% de recettes de l'Etat. Au cours des années 1978,1979\% et 1980, les contributions des entreprises minières s'élevaient à $83,7 \%, 86,8 \%$ de recettes en divises de $1^{\prime} E t a t^{1}$.

Pour régir ce secteur notre pays a déjà connu plusieurs textes légaux commençant par Décret du 16 Décembre 1910, modifié et complété par le Décret du 16 Avril 1919 qui réglementait la recherche et l'exploitation minières uniquement dans le Katanga. Plus tard, cette législation sera remplacée par le Décret du 24 Septembre 1937, pour l'ensemble du Territoire National. Après l'indépendance en 1960, c'est par l'ordonnance-loi n 67/ 231 du 3/ 05/ 1967 portant législation générale sur les mines et les hydrocarbures que l'héritage de la colonisation fut remplacé. L'ordonnance-loi n 81-013 du 2 Avril 1981 portant législation générale sur les mines et les Hydrocarbures a abrogé la précédente, qu'à ce jour, il y a douze ans que la loi $n^{\circ} 007 / 2002$ a été promulgué et mis en application.

Parmi les mérites et les particularités de la loi $\mathrm{n}^{\circ} 007 / 2002$ portant code minier figure la consécration de l'existence légale sur l'exploitation artisanale des mines à travers les dispositions des articles 109 à 128 . La même loi organise les droits miniers qui sont : le permis

1 USELE MAWISA, cité par MUKENDI WAFWANA Emery., Droit Minier Vol.I Principes de gestion du domaine minier, Juricongo, Bruylant, $2 \mathrm{OO}$ pge 43. 
de recherche, le permis d'exploitation, le permis d'exploitation de rejets et le permis d'exploitation à petite échelle; les quels sont constatés par des titres respectifs : certificat de recherche, le certificat d'exploitation, le certificat d'exploitation à petite échelle et le certificat d'exploitation des rejets.

La ville de Kolwezi étant bâtie sur un territoire à richesse minière, elle vit du jour au jour, les réalités juridiques telles que : l'implantation des sociétés commerciales exerçant l'une ou l'autre droit minier: la recherche, l'extraction, le traitement et la commercialisation de substances minières issues de l'exploitation industrielle et artisanale. Bien que régi par la même loi, l'exploitation minière industrielle et l'exploitation minière artisanale n'ont pas les mêmes prérogatives, les mêmes devoirs et les mêmes conditions d'éligibilité. Leurs caractéristiques démontrent qu'ils ne peuvent pas se superposer ni s'empiéter, mais à Kolwezi nous constatons que l'exploitation minière semble présenter un autre aspect particulier de choses eu égard à la volonté du législateur. Et cela engendre plusieurs conséquences néfastes conduisant la présente étude à relever les effets cette dualité : "l'exploitation industrielle et artisanale sur une même concession ». L'exploitation minière artisanale bien qu'étant plus ancienne que l'exploitation minière industrielle, elle semble s'exercer en marge de la loi auvu et su de toutes les autorités tant administratives que juridictionnelles, c'est pourquoi, il nous est loisible de faire état de lieu avant de ressortir les questions épineuses pour y suggérer des pistes de solutions dans la présente réflexion.

\section{ETAT DE LIEU DE L'EXPLOITATION MINIERE INDUSTRIELLE ET ARTISANALE A KOLWEZI FACE A LA LOI 007/2002 PORTANT CODE MINIER}

\subsection{Présentation de la ville de Kolwezi}

Kolwezi est une ville minière de la Province du Katanga, en RDC. Elle est située sur le plateau de la Manika dans le Congo méridional aux coordonnées géographique d'un point de $26^{\circ}$ longitude Est et $11^{\circ}$ latitude Sud. Plusieurs personnes qualifient la ville de Kolwezi de "poumon économique » de la RDC car elle renferme des riches gisements miniers du Cooper Bell. La ville de Kolwezi a une histoire liée intimement à celle de l'union Minière du Haut-Katanga/ GCM et du CSK dans la région du Lualaba ${ }^{2}$.

Construite comme cité ouvrière en 1937, Kolwezi était vaste de 102 ha, érigée à proximité de la carrière de Kolwezi, c'était l'un des premiers camps abritant les travailleurs de la carrière de Musonoie. Plus tard, avec l'ouverture de la carrière de Kamoto, l'union Minière créa la cité ouvrière de Musonoie.

Avec le rattachement à l'union Minière, en 1948, de l'usine de Métalkat, une usine de production de zinc, l'union Minière construisit en 1953, une autre cité ouvrière dénommée cité de Métalkat (à ce jour UZK) d'une superficie de 34 ha. La cité de Ruwe fut lotie et érigée en cité en 1956 (à ce jour cité MUTOSHI).

2 S. Decrée et T. De Putter, Le secteur minier de la RDC à la croisée des chemins, Tervuren, 2010. Page 3. 
En 1953, les activités de l'union Minière l'obligèrent à installer une usine d'électrolyse non loin de la rivière Luilu et à construire de ce fait en 1958, la cité ouvrière de Luilu, ayant un espace de 110 ha, la cité Kapata est la dernière à construire en 1962 pour une étendue de 90 ha.

Le statut de " ville Kolwezi" est effective par l'ordonnance $n^{\circ} 71-177$ du 23 juillet 1976, rattachant à l'ancien centre extra coutumier les territoires de Mutshatsha et de Lubudi pour être urbano-rural en ayant deux Communes urbaines : Manika et Dilala. Le District urbano-rural de Kolwezi est limité au Nord par le District du Haut-Lomami; au Sud-Est par la République de la Zambie et le District du Haut-Katanga; au Sud-Ouest par le District du Lualaba.

Le District-urbano-rural de Kolwezi est baigné par le fleuve Congo (appelé LUALABA) et les lacs de retenue de Kando, N'zilo et de Dikolongo. Avec une superficie de 36.933 $\mathrm{Km}^{2}$, le Territoire de Lubudi $17.861 \mathrm{Km}^{2}$ et le territoire de Mutshatsha : $18.859 \mathrm{Km}^{2}$.

Sur le plan démographique, Kolwezi est habitée par une population qui affiche un taux de croissance continue et presque ininterrompue. Les activités minières industrielles et artisanales sont à la base de la croissance de la population car le marché d'emploi qu'offre différentes sociétés minières attire la crème intellectuelle de différentes villes. Et les jeunes désœuvrés venant de différentes contrées viennent exploiter artisanalement; parfois les différents mouvements échappent au contrôle des autorités mais selon le site internet officiel, cette ville urbano-rurale de Kolwezi compte dans son ensemble 858.538 habitants : la Commune de Manika 197.743 Territoire de Lubudi 366.694 habitants, Territoire de Mutshatsha 167.653 habitants. En plus les étrangers de différentes nationalités sont identifiables. Parmi eux figurent : les chinois, les Belges, les Français, les Libanais, les Indiens, les Brésiliens, les Américains, les Australiens, les Grecs, les Indonésiens, les Britanniques, Zimbabwéens, les Sud-Africains, les Péruviens, les Canadiens, les Marocains... à ce jour, eu égard aux dispositions de l'article 3 de la constitution du 18 février 2OO6, tel que modifiée à ce jour et l'article 5 de la loi organique $n^{\circ} 08 / 016$ du 07 octobre 2008 portant composition, organisation et fonctionnement des entités territoriales décentralisées et leur rapports avec l'Etat et les provinces, la Ville de Kolwezi a le statut d'une entité territoriale décentralisée et tend à devenir un chef-lieu de la future province du Lualaba.

\subsection{Les droits d'exploitation minière industrielle}

Disons avec le Professeur BAKANDEJA que le code de 2002 organise deux types de droits : les droits miniers et les droits de carrière. Les droits miniers sont définis dans les dispositions des articles 50 à 108 pour une exploitation à des fins industrielles et dans celles des articles 109 à 128 pour l'exploitation artisanale. $\left({ }^{3} 1\right)$

Quatre types de droits miniers sont accordés :

$3 \circ$ BAKANDELA WA MPUNGU GREGOIRE, Droit Minier et des Hydrocarbures en Afrique Centrale, éd. Larcier, Afrique édition, Bruxelles 2009 page. 98. 
- Le permis de recherche

- Le permis d'exploitation

- Le permis d'exploitation des rejets

- Le permis d'exploitation à petite échelle.

Ces droits revêtent les caractéristiques de droits réels, de droits immobiliers, des droits exclusifs, de droits cessibles de droits transmissibles et des droits amodiables à l'exception du permis de recherche.

La nature exclusive reconnue aux droits miniers a pour effet d'éviter des empiétements et de superposition des périmètres miniers sauf permission prévue par la loi. A cela les empiètements des périmètres miniers ou des carrières sont réglés soit par le rejet de la demande, soit par la correction de la demande, comme il est écrit : «lorsqu'une demande de droits miniers et / ou de carrières porte sur un périmètre dont plus de $25 \%$ empiètent sur un autre périmètre minier ou de carrières en cours de validité ou est introduit pendant qu'une autre demande est en instruction, cette demande est rejetée $\gg .{ }^{4}$ la situation est corrigée de façon à éliminer les empiètements. Certes par la soustraction du carré empiétant la demande en cause.

En plus de l'empiètement, la loi interdit les superpositions pour la confirmation du caractère exclusif de droits miniers vis-à-vis des droits de carrières et les zones d'exploitation artisanales. Néanmoins l'article $30 \mathrm{du}$ code minier donne une gamme de règles permettant la superposition des périmètres miniers :

$1^{\circ}$ il est permis que le périmètre d'un permis de recherches se superpose sur le périmètre d'une autorisation de recherches des produits de carrières ou d'une autorisation d'exploitation des carrières temporaire (1) Maître MUKENDI WA FWANA justifie que cette superposition est d'autant permise dans la mesure où la recherche des produits des carrières ne devait pas empêcher celle des substances classées en mines qui du point de vue économique, ont une incidence certaine dans la vie économique et sociale du pays $(2)^{5}$. Estimant que l'exploitation des carrières temporaires est d'une courte durée et non renouvelable, ne doit pas empêcher la recherche des substances minérales classées en mines. C'est pourquoi, le périmètre d'un permis des recherches ne peut se superposer sur un périmètre d'un permis d'exploitation, d'un permis d'exploitation de rejet, d'un permis d'exploitation de la petite mine, d'un permis de recherches, d'une autorisation d'exploitation de carrière permanente d'une zone d'exploitation artisanale. Néanmoins, en ver tu de dispositions de l'article 109 alinéa 4 du code Minier, il est permis exceptionnellement l'octroi d'un permis de recherches dans une zone d'exploitation artisanale uniquement pour le groupement d'exploitants artisanaux

$2^{\circ}$ la superposition d'un droit minier d'exploitation sur le périmètre d'une autorisation de recherche des produits des carrières de rechercheest également permise pour des raisons sus vantée. La partie du périmètre d'un droit de recherches des produits de carrière sur

4 Art. $4 \mathrm{O}$ litera a de la loi $\mathrm{N} 0 \mathrm{OO} / 2 \mathrm{OO} 2$ portant code minier.

5 Art. 30 litera a de la loi ${ }^{\circ} 007 / 2002$ portant code Minier. 
laquelle le périmètre d'un droit minier d'exploitation est superposé, est éteinte d'office de ce fait.

$3^{\circ}$ l'on tolère la superposition d'une autorisation de recherches des produits de carrières sur le périmètre d'un permis de recherches.

$4^{\circ}$ le droit des carrières d'exploitation peut se superposer sur le périmètre d'un permis de recherches sorti avec le consentement du titulaire soit par décision du Ministre sur une partie du périmètre d'un permis d'exploitation (1).

Dans la pratique la superposition d'un droit de carrière d'exploitation sur le périmètre d'un permis de recherches connait deux réserves : elle ne peut qu'être partielle et qu'elle doit se faire avec le consentement du titulaire. A défaut de son consentement, par décision du Ministre.

nous pouvons dire qu'en dehors de ces quatre cas de superposition permise, toutes les autres superpositions sont interdites.

\subsubsection{Le permis de recherche}

C'est celui qui confère à son titulaire le droit exclusif d'effectuer, à l'intérieur du périmètre sur lequel il est établi et pendant la durée de sa validité, les travaux de recherche pour lesquelles le permis est accordé et les substances associées en mines pour lesquelles le permis est accordé et les substances associées si le titulairedemande l'extension du périmètre à ces substances $\left(2^{6}\right)$.

Le titulaire d'un permis de recherche est autorisé à prélever des échantillons des substances minérales dans le périmètre faisant l'objet de son permis de recherches pour des analyses ou des assai industriels dans les laboratoires ou dans l'usine de son choix.

Le caractère exclusif du permis de recherche se justifie en plus, lorsqu'un périmètre fait l'objet d'un permis de recherches, aucune autre demande de droit minier pour tout ou partie de ce périmètre ne peut être instruite, hormis la demande de permis d'exploitation du titulaire dudit permis de recherche qui est constaté par un titre appelé : " certificat de recherche ».

Le permis de recherches est un droit temporaire car sa durée est de :

Quatre ans renouvelables deux fois pour une période de deux ans à chaque renouvellement pour les pierres précieuses. Cinq ans renouvelables deux fois pour une durée de cinq ans à chaque renouvellement pour les substances minérales.

Sur le plan de la superficie de périmètre sur lequel peut porter le permis de recherche, il ne doit pas dépasser $400 \mathrm{Km}^{2}$, et une personne et ses sociétés affiliées ne peuvent détenir

6 1. Art. 30 litera a de la loi ${ }^{\circ} 007 / 2002$ portant code Minier

2. BAKANDEJA WA MPUNGU Grégoire., opcit Page. 99

3. Art. 50 al. 2 de la loi ${ }^{\circ} 007 / 2002$ portant code Minier

4. Art. 64 al. 1 de la loi ${ }^{\circ} 007 / 2002$ portant code Minier. 
plus de cinquante permis de recherches (c.-à-d. ne peut dépasser $200.000 \mathrm{Km}^{2}$ sur l'ensemble du territoire).

Le permis de recherche confère un droit d'option au permis d'exploitation.

\subsubsection{Le permis d'exploitation}

C'est celui qui confère à son titulaire le droit exclusif d'effectuer, à l'intérieur du périmètre sur lequel il est établi et pendant la durée de sa validité les travaux de recherche, de développement, de construction et d'exploitation visant les substances minérales pour lesquelles le permis est établi et les substances minérales pour lesquelles le permis est établi et les substances associées s'il en a demandé l'extension. (2)

Le titulaire d'un permis d'exploitation dispose de plusieurs prérogatives :

- De construire et ériger des installations et infrastructures nécessaires à ses activités minières,

- de faire de travaux d'extension de la mine

- De faire louer ou amodier la portion ou toute l'étendue de sa concession à un autre exploitant.

- D'exploiter les gisements artificiels situés dans son périmètre sauf si elle a été exclue expressément

- De faire toute les opérations et techniques de traitement métallurgiques, de concentration

- De poser tous les actes disposition sur les produits marchands extraits, les transporter et commercialiser

- D'user du bois et d'eau trouvés sur sa superficie pour les besoins de l'exploitation minière

Le permis d'exploitation se constate par un titre appelé "certificat d'exploitation" il confère un droit exclusif, cessible, amodiable, transmissible. Sa durée est de trente ans renouvelable plusieurs fois pour une durée de quinze ans.

La superficie du périmètre faisant l'objet du permis d'exploitation est celle du permis de recherches dont il découle ou celle de la partie du périmètre du permisde recherches transformée en permis d'exploitation ${ }^{7}$. Dans les limites de sa concession, le titulaire d'un permis d'exploitation a droit d'exploiter les substances minérales pour lesquelles il est spécifiquement établi. Ces substances minérales sont celles que le titulaire a identifiées et dont il a démontré l'existence d'un gisement économiquement exploitable. Pour exploiter les autres substances associées ou non-associées, le titulaire est tenu d'obtenir l'autorisation d'extension.

Le permis d'exploitation exige de son titulaire la disposition de moyens financiers et matériels suffisamment important pour être en mesure de réaliser ses activités. A cet effet l'État congolais se voit aussi plus exigent à son égard car parmi les conditions d'octroi, figure celle relative à la cession de $5 \%$ des parts du capital social de la société requérante à

7 Art. 68 de la loi $\mathrm{N}^{\circ} 007 / 2002$ portant code Minier. 
l'Etat. Et ces parts sont libres de toutes charges et non diluables, ce qui atteste une véritable intervention de l'Etat que certains qualifient d'ingérence de l'Etat dans les affaires privées des investisseurs.

\subsection{3. le Permis d'exploitation des rejets des mines}

Ce droit minier confère à son titulaire le pouvoir d'exploiter un gisement artificiel qui n'est d'autre qu'une concentration artificielle des substances minérales à la surface provenant de l'exploitation des mines et ou des rejets découlant des traitements minéralurgique et métallurgique : il est certain qu'un gisement artificiel se trouve sur un périmètre couverte par un permis d'exploitation qui exclue cette exploitation, cela peut conduire le requérant à négocier avec le titulaire de périmètre pour une cession de droit d'exploitation de ce gite artificiel, et dans cette hypothèse, il solliciter à la transformation partielle de son permis d'exploitation en permis d'exploitation des rejets de mines ainsi que le transfert de ce permis au cessionnaire. Tandis que sur un gisement qui ne fait pas l'objet de l'exploitation, le Ministre des Mines peut également octroyer un permis d'exploitation des rejets.

Le permis d'exploitation de rejet confère les mêmes prérogatives que dispose le titulaire du permis d'exploitation, seulement il ne peut pas exploiter en profondeur, car son droit se limite à la surface que couvre le gite artificiel. Sa durée est de cinq ans renouvelable plusieurs fois pour la même durée. Et le titre qui constate ce droit est le certificat d'exploitation de rejet. (1)

\subsubsection{Le permis d'exploitation minière à petite échelle}

Le permis d'exploitation minière à petite échelle est un droit réel immobilier, exclusif, cessible, amodiable et transmissible droit qui est constaté par un titre minier dénommé « certificat d'exploitation de petite mine. » les articles 97 à 108 de la loi n 007/2002 fixent les règles et modalités relatives à l'exploitation minière à petite échelle. (2)

Ce permis est accordé lorsque les conditions techniques caractérisant certains gisements de substances minérales ne permettent pas d'en faire une exploitation à grande échelle économiquement rentable mais permettent une exploitation minière de petite taille avec un minimum d'installations fixes utilisant des procédés semi-industriels ou industriels;

Le titulaire d'un permis d'exploitation minière à petite échelle a un droit exclusif d'effectuer à l'intérieur du périmètre octroyé les travaux de recherche, de développement, de construction et d'exploitation des substances minérales sur lesquelles il porte sa durée est variable, mais ne peut dépasser dix ans, y compris les renouvellements. Il est écrit que "toute fois", moyennant l'avis de direction de mines, le ministre peut proroger le permis de l'exploitation de petite mine le cas et le pour les substances dont l'exploitation dépasse dix ans.(1) Le permis d'exploitation de petite mine confère à son titulaire le droit d'exploiter les substances minérales pour lesquelles il est spécialement établi et dont le titulaire a identifié 
et démontré l'existence d'un gisement. Il peut s'étendre aux substances associées ou non associées. $(2)^{8}$

Tous les droits miniers que nous venons de citer sont soumis aux conditions d'éligibilités de leurs requérants ainsi que les obligations liées à l'exercice de ces droits. ${ }^{9}$

\section{Conditions d'éligibilités aux droits miniers}

L'état qui exerce la souveraineté permanente sur le sol et le sous-sol qui renferme les gites minéraux, les cours d'eau confie la prospection, la recherche et l'exploitation minière à toute personne physique ou morale de son choix, qui peut répondre aux critères définis par les dispositions de l'article 27 de la loi $n^{\circ} 007 / 2002$ portant code minier, qui garantit un principe de liberté en permettant à toute personne physique ou morale de nationalité congolaise ou étrangère ainsi les organismes à vocation scientifique de solliciter un droit minier. Ce principe de liberté d'accès connait des restrictions dans le but soit de protection de la personne qui s'engage, c'est le cas des incapables (les mineurs d'âge par exemple), les interdits de ceux qui exercent certaines professions incompatibles (c'est le cas de fonctionnaires de l'état et agents, les éléments de la police nationale congolaise et de la FARDC).

Au sujet de personnes morales, l'article 23 all du code minier pose à titre de principe que les personnes morales de droit congolais ayant leur siège social et administratif dans le territoire national et dont l'objet porte sur les activités minières sont seules éligibles aux droits miniers. Ces personnes morales peuvent être de droit public (province, entité territoriale décentralisée, entreprise publique) ou de droit privé (sociétés commerciales, associations sans but lucratif) qui doivent exister en conformité de la législation congolaise.

Obligations relatives aux droits miniers

Au-delà des obligations administratives, les titulaires de droits miniers sont soumis aux obligations de la protection de l'environnement. C'est ainsi qu'avant de commencer les travaux de recherche miniers, le titulaire d'un permis de recherches doit élaborer et obtenir l'approbation d'un plan d'atténuation et de réhabilitation (PAR) pour l'activité proposée.(1)

Aussi, le tout demandeur d'un permis d'exploitation, de rejets, de petite mine est tenu de présenter une étude d'impact environnemental (EIE) accompagnée d'un plan de gestion environnementale du projet (PGEP) et d'en obtenir l'approbation. Etant donné que les activités minières détériorent d'une manière directe ou indirecte l'écosystème, la flore : la nature.

C'est pourquoi cette étude d'impact environnemental doit présenter une description de l'écosystème avant les opérations, y compris la faune, la flore, les sols et la topographie, la

8 1. Art. 101 al 2 de la loi $n^{\circ} 007 / 2002$ portant code minier

2. BAKANDEJA WA MPUNGU Grégoire; op cit page 116.

9 Art. 203 de la loi $n^{\circ} 007 / 2002$ portant code minier. 
qualité de l'air, des eaux souterraines et de surface, à cela, il faut y ajouter les mesures envisagées pour la protection de l'environnement, la limitation des pollutions, et la reconstitution des sites ainsi que vérifier l'efficacité de ces mesures.

La ville de Kolwezi renferme plusieurs entreprises minières, plusieurs d'entre elles sont en partenariat avec la GECAMINES. Sans faire une liste limitative nous pouvons citer les plus visibles :

- Générale de carrières et mines (GECAMINES);

- Kamoto Copper Company (KCC);

- Tenke fungurume mining (TFM);

- Mutanda mining (MUMI);

- Boss mining;

- Chemical of africa (CHEMAF);

- Société minière de Kolwezi (SMK)

- Anvil mining company of Katanga (AMCK)

- Kisanfu mining (KIMIN);

- La sino congolaise des mines;

- Kansuki mining;

- Volcano, SWANEMINES, COMIDE, ROCK MINES...

Ces entreprises produisent plus de cuivre et du cobalt mais, la ville de Kolwezi renferme d'impact gisements de cuivre à haute teneur et des minerais associés tels que : le zinc, le plomb, l'argent, l'uranium, le rhénium, le nickel, certes les activités de ces entreprises minières ont un impact remarquable sur plusieurs plans socio-économiques de population.

\subsection{L'EXPLOITATION MINIERE ARTISANALE}

Elle est régie par les dispositions des articles 109 à 128 du code minier qui organisent non seulement l'institution des zones d'exploitation artisanale, mais aussi la commercialisation, la transformation et l'encadrement des exploitants artisanaux que nous appelons "creuseurs".

Quant à l'institution des zones d'exploitation artisanale, cela n'est possible que lorsque les facteurs techniques et économiques qui caractérisent certains gites d'or, de diamant ou de toute autre substance minérale ne permettent pas d'assurer une exploitation industrielle ou semi-industrielle. L'institution d'une zone d'exploitation artisanale est faite par voie d'arrêté du ministre ayant les mines dans ses attributions après avis de la direction des mines et du gouverneur de la province concernée. (1) la loi interdit de procéder à l'exploitation artisanale dans un périmètre couvert par un titre minier en cours de validité, de même aucun titre minier ne peut être octroyé aux tiers, tant que la zone d'exploitation artisanale existe.

En respectant la théorie du parallélisme juridique, le même ministre peut procéder à la fermeture d'une zone à exploitation artisanale lorsqu'on y découvre un gisement qui demande une exploitation industrielle et cela suite aux travaux de prospection et de recherches 
de la de la direction de géologie ou du groupement des exploitants miniers artisanaux qui ont un droit de préemption.

L'exploitation artisanale étant l'activité par laquelle une personne physique de nationalité congolaise se livre dans une zone d'exploitation artisanale délimitée en surface et en profondeur jusqu'à trente mètre au maximum, à extraire et à concentrer des substances minérales en utilisant des outils, des méthodes et des procédés non industriels. (2)

De cette définition, nous pouvons dire que l'exercice de la profession d'exploitant artisanal est subordonné à la détention d'une carte d'exploitant artisanal délivrée par le chef de division provinciale des mines, aux personnes physiques, majeures d'âge de nationalité congolaise. ${ }^{10}$

Dans L'intérêt de la protection de l'environnement, les exploitants artisanaux sont tenus de respecter le code de bonne conduite de l'exploitation artisanale et de contribuer au "fonds de réhabilitation" en vue de financer la réalisation des mesures d'atténuation et de réhabilitation des zones d'exploitation artisanale.

La carte d'exploitation artisanale a une validité d'une année, renouvelable pour la même durée sans limitation. Elle procure la liberté de détenir, et de commercialiser et de transporter les produits de l'exploitation artisanale des substances minérales. L'article 116 du code minier dispose que "les exploitants artisanaux ne peuvent vendre leur produits miniers qu'aux négociants, aux marchés boursiers, au comptoir agrée”.

Les exploitants miniers artisanaux sont soumis à l'encadrement technique assuré par les services spécialisés relevant du ministère des mines (SAESCAM) et les coopératives doivent apporter assistance dans l'accomplissement de toutes les formalités. Aussi cet encadrement doit porter sur les manières de respecter le code de bonne conduite environnemental, des règles de l'art et de l'hygiène. L'acquisition et l'utilisation des équipements de recherche et de transformation adaptés au type de gisement et de substances minérales.

Au regard de toute la législation sur l'exploitation minière artisanale, il est certain que le district urbano-rural de Kolwezi est caractérisé par une exploitation minière artisanale portant, non seulement sur les pierres précieuses mais surtout aux produits hétérogénéites, car depuis l'indépendance de notre pays jusqu'à l'effondrement du secteur minier du Katanga en général, a longtemps puisé ses ressources financières dans l'exploitation industrielle des mines de cuivre. Avec cette chute de l'industrie minière qui a entrainé l'effondrement économique engendrant les chômages et la fermeture des petites et moyennes entreprises gravitant autour de la Gécamines.

Et la ville de Kolwezi était au centre de tout le grand symptôme de cette chute qui était aggravée par plusieurs évènements dont les plus remarquables étaient :

- Les grèves des travailleurs de mois de mai et juin 1990

- L'effondrement ou éboulement de la mine souterraine de Kamoto

10 1. BAKANDEJA WA MPUNGU, op cit pge. 116

2. Art 1 litera 21 de la loi $n^{\circ} 007 / 2002$. 
- Les perturbations d'origine politique conduisant au refoulement des originaires de deux provinces du Kasaï

- L'augmentation de charges financières liées à la commercialisation et l'endettement à court terme très élevé de la Gécamines...

Cette crise a été à la base de plusieurs problèmes sécuritaire, car les vols caractérisés par plusieurs dénominations (opération, mitraille, frappe) ont d'avantage rongé la géante GECAMINES, que vers 1994, l'exploitation de l'or par les creuseurs a semblé tant soi peu apporter un petit redressement et de la vie sociale. Cette activité a vu être règlementée par la loi de 2002 portant code minier.

Attendu que depuis la mise en vigueur de la loi $n^{\circ} 007 / 2002$ du 11 juillet 2002 portant code minier, le 11 janvier 2003 jusqu'à ce jour, l'exploitation minière artisanale n'est pas à l'abri de critiques tant positives et négatives. Dès lors l'exploitation minière industrielle et artisanale dans la ville de Kolwezi reflète plusieurs problèmes qui méritent d'être relevés dans cette partie de notre travail.

\section{PROBLEMES RELATIFS A LEXPLOITATION MINIERE INDUSTRIELLE ET ARTISANALE A KOLWEZI}

A la mise en vigueur de la loi $n^{\circ} 007 / 2002$ portant code minier, l'exploitation minière artisanale était effective, mais le gouvernement n'a pas institué plusieurs zones à exploitation minière artisanale en vue de permettre les exploitants artisanaux à exercer leurs activités en toute légalité. Mais il s'est institué une pratique consistant à exercer et à utiliser les creuseurs dans les concessions couvertes par les droits miniers. Il s'agit de la superposition de l'exploitation artisanale sur les périmètres couverts par les permis de recherches et permis d'exploitation. Cette superposition engendre plusieurs conséquences sur plusieurs plans; juridique et judiciaire, de l'environnement et l'habitat, sécuritaire d'investissement des grandes entreprises, démographique...

a) De la superposition de l'exploitation minière artisanale sur les droits miniers industriels

De manière générale, il est inconcevable d'admettre la superposition du droit minier au droit foncier car un seul espace ne peut pas abriter à lui seul les droits de nature foncière et de nature minière, il y a exclusion entre les deux droits) ${ }^{11}$. Comme nous l'avons relevé précédemment sur un périmètre couvert peut pas y instituer une zone a exploitation artisanale.

Attendu qu'en dépit du caractère exclusif de droits miniers, les articles 30 et 40 du code minier interdit tout empiètement ou toute superposition sauf dans les cas limitatifs par le législateur, mais dans la ville de Kolwezi, les activités minières artisanales qui sont plus remarquables et fructueuses pour les acteurs sont celles exercées dans les concessions couvertes par les titres miniers et cela pour plusieurs raisons dont les principales sont :

11 10. Adolphe BAMBI KABASHI; le droit minier congolais a l'épreuve des droits fonciers et forestiers, le harmattan RDC, paris, 2002? page 117. 
- Manque ou insuffisance de zones à exploitation artisanale instituée par le ministre de mine;

- La recherche de la bonne teneur de produits miniers qu'on trouve dans les concessions couvertes par les titres miniers qu'ailleurs;

- La facilité d'exploiter offerte dans les concessions couverte par des titres miniers par rapport au dur labeur de découverture;

- La volonté de titulaire de droit minier qui parfois manquent des moyens financiers nécessaires à exploiter industriellement leurs concessions...

\section{A.1. Manque et insuffisance de zones à exploitation artisanale dans la ville de Kolwezi}

Il s'avère que même la carrière de Kawama qui regorge un nombre élevé de creuseurs est une concession couverte par un titre minier. C'est suite aux troubles et manifestations de creuseurs que les autorités provinciales avaient demandé aux exploitants artisanaux de continuer leurs activités en attendant une solution définitive, et une autre concession considérée comme zone à exploitation artisanale serait même à l'ignorance de concernés. C'est pourquoi les exploitants miniers artisanaux sont souvent dans les concessions appartenant à autrui où ils sont chaque fois chassés, déguerpi et leurs produits confisqués. A cela, selon certains responsables de coopératives minières, il faut ajouter le fait que bon nombre de périmètre miniers qui sont aux alentours de la ville ou des villages du district de Kolwezi soient visité par les clandestins en dépit des zones à exploitation artisanale qui sont située loin des agglomérations voire inaccessibles.

Il est à noter que la présence de comptoir d'achat de produits miniers à cote de la ville ou dans les cités, encourage d'avantage l'exploitation minière artisanale dans la mesure où le circuit de la commercialisation tel que prévue par la loi n'est pas respecté, car les creuseurs vendent directement leur produits miniers sans passer par les négociants.

A ce jour il nous semble difficile de dénombrer les exploitants miniers artisanaux à Kolwezi à cause du caractère informel de cette activité.

\section{A.2. La recherche de la bonne teneur en cuivre et cobalt}

Actuellement l'exploitation minière artisanale dans la ville de Kolwezi, recherche le cuivre et le cobalt dans les produits hétérogénéités dont la qualité s'apprécie par rapport à la teneur élevée de produits vendus qui dans la plus part sont plus riches en teneur, les produits issus de concession minière couverte par de titre mais les produits de zones à exploitation artisanale sont à faible teneur et cette situation pousse les creuseurs à abandonner leur zone à exploitation artisanale vers les concessions couverte par les titres miniers. 


\section{A.3. La facilité de travail dans les concessions minières couverte par des titres miniers}

Les exploitants miniers artisanaux préfèrent travailler dans les concessions où les travaux de la découverte avait déjà été fait par les engins, plutôt dans zone à exploitation artisanale ou ils doivent par leur force manuelle aux moyens et instruments rudimentaires faire la découverture avant toute exploitation, plutôt aller là où le travail est facilité : dans les anciennes remblaies ou de nouvelles découvertures qui sont aux environs de la ville.

\section{A.4. La volonté de titulaires de droits miniers}

Certains détenteurs de titre minier permettent l'exploitation artisanale sur leurs sites en contrepartie d'un pourcentage de la production et cela en violation de la loi. Cette pratique qui a aidé la Gécamines et ses partenaires de l'époque tel que : Groupe BAZANO, SOMI$\mathrm{KA}, \mathrm{CHEMAF}, \mathrm{EGMF} .$. à ce jour certaines d'entre elles se conforment à la loi, mais les entreprises comme SOCOMINES et CHEMAF continuent de traiter avec les artisanaux. D'une autre part cette pratique bien qu' illégale, elle figurait parmi les voies de solutions contre les vols de substance minérales dont étaient victimes certains titulaires de droit minier, que les creuseurs reprochaient aussi dans la réduction de leur teneur de poids, d'humidité, et d'analyse tronquée dans le but de les exploiter, étant donné que ces creuseurs n'ont pas droit d'aller vendre leur produits ailleurs, cela avait fait l'objet d'un constat de l'Amnesty international. En juin 2013 qui estimait qu'il y a 70.000 et 150.000 exploitants artisanaux dans la province du Katanga, bien que certaines informations indiquent que le nombre diminue a fur et à mesure que l'exploitation minière industrielle prend de l'importance. Les creuseurs sont exploités à la fois par les responsables des pouvoirs publics et les acteurs privés qui contrôlent les sites miniers. Ils sont souvent obligés de vendre leurs produits minéraux extraits à des personnes ou à des entreprises déterminées, sous peine de se voir interdire l'accès au site et donc perdre leurs moyens de substances.

L'exploitation artisanale exercée dans les zones appropriée et celle exercée dans les sites couverts par les titres miniers, sont caractérisées par des constats déplorables ci-après :

- Les conditions de travail déplorables et extrêmement dangereuses;

- Les creuseurs travaillent à main nue, sans équipements de protection adéquats;

- Les puits mal ventilés où la température peut être particulièrement élevée;

- Le sous équipement de sécurité et ils sont exposés aux chutes de pierres, inhalation de poussière...

- Des dizaines de creuseurs meurent ou sont grièvement blessés des effondrements de galeries.

b) Problèmes juridiques et judiciaires de l'exploitation minière artisanale

Sur le plan de droit, la loi $\mathrm{n}^{\circ} 007 / 2002$ portant code minier fait aujourd'hui l'objet de critiques qui conduisent déjà à sa modification, néanmoins il y a lieu de relever que le fait d'étendre l'exploitation artisanale aux minerais qui nécessitent l'implantation des industries 
de traitement et de transformation tel que du cuivre et du cobalt. En procédant ainsi et sachant que les industries de traitement et de transformation ne sont construites que par les entreprises minières qui peuvent ne pas s'intéresser aux minerais artisanaux et tourné vers l'extérieur du pays.

En plus nous dirons avec le professeur TSHIZANGA qu'étant donné que les marchés boursiers, qui en tout de chaines sont habilités à acheter les minerais issus de l'exploitation artisanale ne sont pas encore crées, ce sont les sociétés dénommées "Mining" qui pullulent et dont la plupart ne détiennent ni droits miniers ni usines de traitement et de transformation, qui les achètent à vils prix et les exportent frauduleusement. Par ailleurs, la non création de la zone d'exploitation artisanale riche en teneur incite les creuseurs à violer les dispositions de l'article 109 du code minier, et sous le regard impuissant des titulaires des droits miniers et des autorités du gouvernement qui dans certains cas encadrent même ces creuseurs.

Outre l'obligation de s'engager à respecter la règlementation en matière de protection de l'environnement de l'hygiène et de la sécurité dans les zones d'exploitations artisanales, tel que prévu par l'article 111 al 2 du code minier, il semble que le législateur n'a pas mis un accents particulier sur la protection de l'environnement par les creuseurs, et par manque des dispositions claires, comme il l'a fait avec les exploitants miniers industriels qui sont obligés à produire le plan d'atténuation et de réhabilitation (PAR), étude d'impact environnemental (EIE) et le plan de gestion environnementale (PGEP), les creuseurs laissent les périmètres exploités tels quels après leurs activités.

A la lecture minutieuse de l'article 81 qui dispose que "sous réserve des dispositions prévues à l'article 10. Litera $\mathrm{j}$, le traitement ou une entité de transformation", il nous semble que le législateur habilite les personnes morales ou les personnes physique qui n'ont de permis ni d'exploitation ni d'autre droits miniers à s'adonner aux activités de traitement ou de transformation des minerais provenant des exploitants miniers industriels, et cela dans le souci d'encourager le développement des industries de traitement et de transformation en dehors des usines appartenant aux exploitants miniers, et aussi favoriser la collaboration inter-entreprises sous forme de sous- traitance. Conformément aux dispositions des articles 82 et 83 du code minier, cette activité est subordonnée à l'autorisation de ministre ce qui prête à confusion de compétence entre le ministre de l'environnement et le ministre des mines et ce dernier a compétence d'autoriser la transformation de produits issus de l'exploitation artisanale.

Sur le plan judiciaire, l'exploitation minière artisanale est à la base d'une criminalité non seulement sur le plan de droit commun, mais aussi sur le plan de droit minier. La loi n ○007/2002 portant code minier prévoit des infractions et des pénalités par les dispositions des articles 299 à 311. Dans la ville de Kolwezi, les instances judiciaires connaissent des dossiers en répression. Les tribunaux de paix, le tribunal de grande instance n'en connaissent pas beaucoup car bon nombre de dossiers se clôturent devant les officiers de polices judiciaires et les officiers du ministère public qui fixent des amendes transactionnelles en deçà des valeurs prévues par la loi. C'est le cas de : 
- Vols et des réels des substances minérales dont les amendes vont de l'équivalent de 5000 Dollars à 20000 USD;

- Détention illicite des substances minérales dont l'amende va de 2000 dollars à 20000 USD...

Souvent les personnes poursuivies de ces infractions sont pauvres et dans l'appréciation de la sanction, les officiers du ministre public, perçoivent selon la possibilité de personnes poursuivies.

Cette réalité sur terrain exprime l'irréalisme juridique, qu'une révision s'avère nécessaire pour adapter des sanctions aux réalités sur terrain.

En outre, l'exploitation artisanale est à la base de plusieurs dossiers pénaux, dont les fréquences portent sur les viols faits à la femme et aux filles mineures, il s'avère que les activités périlleuses de galerie communément appelé dans le jargon "Kalolo" poussent les creuseurs à se droguer par toute sorte des stupéfiants, cela est à la base de la criminalité. Sans oublier l'ivresse publique qui semble tomber en désuétude à cause de la tolérance des autorités chargées des recherches des infractions.

\section{c) Problèmes sociaux et environnementaux}

L'exploitation minière artisanale dans la ville Kolwezi est à la base de la transformation d'une partie du quartier résidentielle de Kasulo et Kakifuluwe de la commune de Manika en une carrière et cela en violation des lois et sous l'impuissance des autorités du gouvernement tant provincial que central et cet assaut engendre plusieurs effets néfastes :

- Destruction des infrastructures de l'état; les avenues et rues;

- Destruction de maisons d'habitation;

- La recrudescence des conditions environnementales déplorables, or cette situation a connu une amélioration avec l'exécution de l'arrêté urbain n²012/005 portant fermeture et délocalisation des dépôts d'emmagasinage de produits de l'exploitation minière artisanale vers le site approprié de MUSOMPO, qui a sauvé l'avenue SALONGO de la même commune dans le quartier industriel de la ville de Kolwezi;

- L'insalubrité et la multiplicité des petits commerces informels au bord des avenues sans moindre protection;

- Les décès par effondrement et asphyxions de plusieurs personnes parmi lesquelles figurent les mineurs d'âge;

- La croyance aux pratiques magico-religieuse capable de rehausser la teneur de produits minéraux en cobalt ou cuivre, conduisant leurs auteurs aux infractions de meurtre et mutilation de cadavre. Depuis le début des activités minières artisanales dans le quartier résidentiel de KASULO, du mois de mai 2014 au décembre 2014, l'on a compté un quinzaine de cas de corps sans vie retrouvés le matin avec mutilation soit des organes génitaux, soit de mutilation des organes de sens. 
d) Problème d'insécurité contre les investisseurs industriels

L'exploitation minière artisanale dans la ville de Kolwezi est à la base de plusieurs cas de vols et de destructions méchantes dont sont victimes les grandes sociétés de l'état à l'instar de la GECAMINES, la SNCC et la SNEL.

\section{VICTIME - GECAMINES}

En plus des actes déplorés précédemment qui ont concouru à l'effondrement de la GCM, citons également l'infiltration des creuseurs dans ses différentes sites comme DEZIWA, MUTOSHI...les creuseurs détruisent parfois des repères topographiques tels que les points géodésiques de base avec balise. La Gécamines est en plus victime de destruction des infrastructures dans les mines en veilleuse tels que les pistes, les stations de pompage... Sans oublier la destruction désordonnée de roches ce qui entrainerait des coûts exorbitants lors de la reprise de l'exploitation.

\section{VICTIME - SNCC}

A ce jour, au-delà des problèmes que connait cette grande société qui autre fois facilitait le transport de minerais appartenant à la GECAMINES, chemin faisant les quantités et poussières qui tombaient de wagons après plusieurs années ont formé une couche sur la voie ferrée que les creuseurs ont fait l'assaut pour détruire la voie ferrée, au ramassage de produits cuivreux. Cette situation vient enfoncer d'avantage la misère de la société nationale de chemin de fer qui, à cause de la vétusté de ses matériels connait plusieurs cas d'accident et déraillement de ses trains. Nous ne pouvons pas oublier les cas de destructions méchantes des câbles électriques en cuivre haute tension...

e) Sur le plan démographique et humain

Parmi les problèmes que connait le district urbano-rural de Kolwezi, à la suite de l'exploitation artisanale figuré aussi l'exode de la main du secteur agricole vers le centre urbain. Et cela a un impact sur le nombre des habitants de Kolwezi qui connait une augmentation. La présence de femme et des enfants qui constitue une classe de personnes vulnérables est à la base de plusieurs méfaits tels que :

- Les cas de malformation congénitale sont nombreux à Kasulo qu'ailleurs;

- Les femmes qui autre fois étaient découragées par certains ONG, avec les activités minières de Kasulo, elles sont de nouveau replongées dans les travaux insalubres;

- L'utilisation des enfants mineurs dans lavage, triage, mise en sac, chargement et déchargement de produits miniers communément appelé « salize »

- Le préjudice environnemental dû à une explosion incontrôlée et le non-respect des règles de protection contre les produits radioactifs et pollution de rivières et nappes aquifères. 
f) Le problème d'encadrement des exploitants miniers artisanaux

En autorisant l'exploitation minière artisanale la même loi reconnait à l'Etat le pouvoir de créer seul ou en association avec des tiers se livrer à une activité de régie par le code minier. Par décret n ${ }^{\circ} 047-\mathrm{C} / 2003$ du 28 mars 2003 portant création et statut d'un service public dénommé service d'assistance et d'encadrement du small scale mining ou production minière à petite échelle.

Le SAESCAM est un service public à caractère technique, doté d'une autonomie administrative et financière qui a pour objet :

- La promotion d'une classe moyenne congolaise dans le secteur de la petite mine en assurant la formation et en apportant l'assistance technique et financière aux coopératives minières et aux exploitants du secteur de la petite mine, en vue de renforcer leurs capacités managériales;

- Inciter le regroupement des exploitants miniers artisanaux en coopératives; en les encourageant à l'acquittement de leurs obligations;

- Contribuer à l'amélioration du bien-être des communautés locales ou se déroulent les activités minières artisanales...

Certes dans la ville de Kolwezi ce service existe, ces efforts fournis pour l'encadrer les creuseurs semblent ne pas être suffisant à cause de plusieurs difficultés tant sur le plan logistiques que financiers. Il semble que l'Etat ne pourvoit pas de moyens capables de répondre aux besoins du bon fonctionnement voire l'existence de certaines coopératives minières ne sont que de fait, hormis quelques-unes comme l'EMAK qui autre fois a brillé mais à ce jour les membres semblent être visibles dans l'encadrement des exploitants miniers artisanaux qui travaillent en informel.

\section{SUGGESTIONS AUX PROBLEMES D'EXPLOITATION MINERE INDUSTRIELLE ET ARTISANALE DANS LA VILLE DE KOLWEZI}

Il est vrai que l'exploitation minière artisanale à Kolwezi figure parmi les vielles activités qui jadis qu'on appelait "lwanzo Lwa mikuba" étant une activité des initiés, elle a précédé l'exploitation minière industrielle. Eu égard aux difficultés et conséquences dû aux empiètements et superposition de ces deux activités qui du reste sont incompatibles, nous pouvons proposer ce qui suit :

- Une mise en place d'une autorité régulateur en mine et d'un véritable " état de droit";

- La révision de la loi $n^{\circ} 007 / 2002$ portant code minier, pour permettre au ministre des mines la compétence d'instituer même sur un gisement qui peut nécessiter l'exploitation minière industrielle une zone à exploitation minière artisanale et améliorer d'autres dispositions du code minier;

- Que 1'Etat congolais applique la décentralisation technique du cadastre minier; 
- La création d'une option ou centre de formation en mine artisanale pour l'encadrement de la jeunesse à l'exploitation minière;

- La formation des agents de l'Etat du secteur minier au respect de la déontologie.

\section{CONCLUSION}

Ce travail est constitué pendant que les méfaits de l'exploitation minière artisanale ravagent encore la ville de Kolwezi. Certes, les statistiques de cas concrets vécu sont encore en voie de faire l'objet du bilan. L'activité minière artisanale a des avantages sur le plan socio-économique des habitants de la ville de Kolwezi, mais sur le plan national, elle semble être une activité qui ne rapporte presque rien au budget national. Pour preuve, en lisant le rapport ITIE-RDC à travers sa synthèse du 4è rapport, exercice 2011 paru en juillet 2014, il n'y figure aucune recette issue de cette activité. C'est pourquoi, certains proposent sa suppression pour laisser l'exploitation minière industrielle. Pour notre part l'encadrement efficace des exploitants artisanaux est la voie par excellence pour lutter contre toutes les conséquences relevées dans ces recherches, car la traçabilité de produits miniers issus de l'exploitation minière artisanale, génère aussi de recettes au profit de l'État, qui devraient en principe inscrite dans ce compte pour la recherche du bien-être commun. Le gouvernement qui contrôle les activités minières dispose d'une responsabilité de protéger les investisseurs les exploitants artisanaux et la population qui est victime de conséquences des activités minières.

\section{BIBLIOGRAPHIE}

La loi n ${ }^{\circ} 007 / 2002$ portant code minier;

Le décret du 16/12/1910 tel que modifier et compléter par le décret de la 16/08/1910 portant règlementation de la recherche de l'exploitation minière au Katanga;

Code larcier de la RDC, Tome IV droit économique 2002;

MUKENDI WAFWANA Emery., Droit Minier Vol.I Principes de gestion du domaine minier, Juricongo, Bruylant, 2005

BAKANDELA WA MPUNGU GREGOIRE, Droit Minier et des Hydrocarbures en Afrique Centrale, éd. Larcier, Afrique édition, Bruxelles 2009;

Adolphe BAMBI KABASHI; le droit minier congolais à l'épreuve des droits fonciers et forestiers, le harmattan RDC, paris, 2002?

Decrée S. et De Putter T., Le secteur minier de la RDC à la croisée des chemins, Tervuren, 2010. Page 3. 